\title{
Efficient Biometric Iris Recognition Based on Iris Localization Approach
}

\author{
Muzhir Shaban Al-Ani', Salwa Mohammed Nejrs² \\ ${ }^{1}$ Department of Information Technology, University of Human Development, College of Science and Technology, \\ Sulaymaniyah, KRG, Iraq, ${ }^{2}$ Department of Physics, University of Misan, College of Science, Iraq
}

\section{A B S T R A C T}

Biometric recognition is an emerging technology that has attracted more attention in recent years. Biometric is referred to physiological and behavioral characteristics to identify individuals. Iris characteristic is related to physiological biometric characteristics. Iris recognition approaches are among the most accurate biometric technologies with immense potential for use in global security applications. The aim of this research is to implement an efficient approach to process the diseased eye images to verify the second iris examination for the same person by inserting an improvement step to the iris recognition system. The improvement step makes a correction of boundary around the pupil and removes the corrupted regions. This approach demonstrates its ability to detect the inner limit of the iris. The obtained results show that $90 \%$ success in the detection of diseased eye images, which make the iris recognition system more accurate and safe.

Index Terms: Biometric recognition, Iris Localization, Iris Recognition, Template Matching

\section{INTRODUCTION}

Biometric identification is a new technology to recognize a person based on a physiological or behavioral characteristic that attracting a lot of attention recently [1-3]. As the level of counterfeit and deceptive transactions increases rapidly, so this causes the need for highly secure identification technologies and personal verification [4-6]. The existing methods of shared secrets such as PINs or passwords, key devices, and smart cards, these are not sufficient in many applications [7-9]. Biometric characteristic can realize this issue that is unique and realize the characteristic of a human [10-12]. The use of biometrics for personal authentication becomes practical and considerably more accurate than the current methods [13-15].

\begin{tabular}{|l|l|}
\hline \multicolumn{2}{|c|}{ Access this article online } \\
\hline DOI: 10.21928/uhdjst.v3n2y2019.pp24-32 & $\begin{array}{l}\text { E-ISSN: 2521-4217 } \\
\text { P-ISSN: 2521-4209 }\end{array}$ \\
\hline $\begin{array}{l}\text { Copyright @ 2019 Al-Ani and Nejrs. This is an open access article } \\
\text { distributed under the Creative Commons Attribution Non-Commercial } \\
\text { No Derivatives License 4.0 (CC BY-NC-ND 4.0) }\end{array}$ \\
\hline
\end{tabular}

The biometric characteristics are classified into two main categories [16,17]: Physiological characteristics related to the shape or part of the body, such as iris, fingerprint, face, DNA, retina, and the geometry of the hand [18-20]. The behavior characteristics are related to the human behavior, such as gait, voice, signature, and keystroke dynamics [21-23]. Biometrics can be applied in companies, governments, military, border control, hospitals, banks ..., etc. [24-26]. These characteristics are used to verify the identity of a person for allowing access to certain information [27-29].

The most important characteristics of the iris do not change the texture of the iris through a person life [30,31]. This stability of iris features over a long time, leading to guarantees the long period of validity of the data and it does not need to update; in addition, iris characteristics are well protected from the environment [32-34]. This advantage allows iris identification as the most accurate and reliable biometric identification [35-37]. In the entire human population, there is no similarity two irises in their mathematical details, even between identical twins [38-40]. The probability of finding

Corresponding author's e-mail: Muzhir Shaban Al-Ani, Department of Information Technology, University of Human Development, College of Science and Technology, Sulaymaniyah, KRG, Iraq. E-mail: muzhir.al-ani@uhd.edu.iq

Received: 16-05-2019

Accepted: 23-07-2019

Published: 31-07-2019 
two people with an identical iris is almost approach zero, and the probability that two irises are similar; it is approximately 1 in $10^{10}$ [41-43].

Iris recognition is an effective aspect of human identification for its dissimilarity between iris characteristics. These research aims are to introduce an efficient biometric iris recognition approach based on iris localization method. This approach tries to improve the identification process through certain processes on iris image.

\section{IRIS RECOGNITION}

The recognition of the iris is an automatic method of biometric identification that uses mathematical techniques of pattern recognition in video images of one or both irises of an individual's eyes [44,45]. The complex iris patterns are unique, stable, and visible from a distance $[46,47]$. The iris recognition technology determines the identity of an individual through many steps, as shown in Fig. $1[48,49]$. These steps of iris recognition are as follows:

- Iris image acquisition: This step deals with using of electronic devices that converting the object into digital images such as digital camera and digital scanner [50,51].

- Image preprocessing: The iris image is preprocessed to obtain a useful region iris image such as to illustrate the detection of the inner and outer boundaries of the iris. This step detects and removes the eyelids and eyelashes that may cover the eye image [52]. The iris image has low contrast and uneven illumination caused by the position of the light source, so preprocessing try to recover these aspects. All of these factors can be compensated in the image preprocessing step [53].

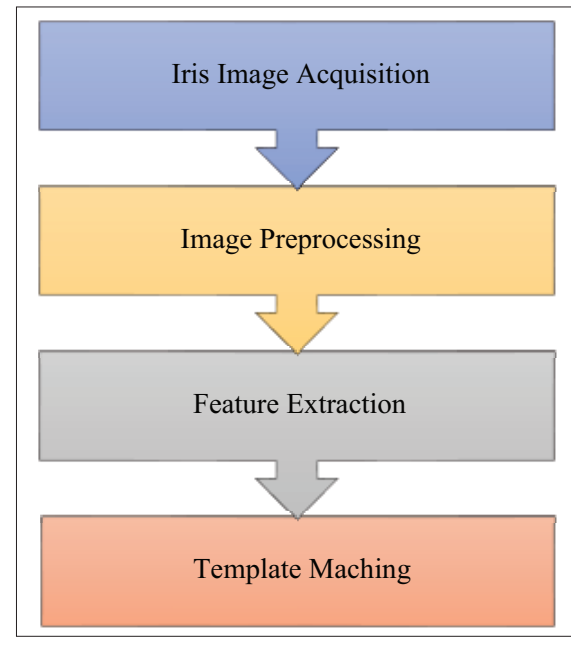

Fig. 1. Iris recognition system.
- Feature extraction: This step deals with generating of features applying the texture analysis method to extract features from the normalized iris image [54]. Important features of the iris are extracted for precise identification purposes [55].

- Template matching: This step deals with comparing the user model with the database models using a corresponding matching statistic [56]. The corresponding metric will give a measure of similarity between two iris models or template. It provides a range of values when comparing models of the same iris and another range of values when comparing different iris models [57]. Finally, a high confidence decision is made to identify whether the user is authenticated or not [58].

\section{LITERATURE REVIEW}

Many literature reviews are published related to iris recognition. This section introduces some of the updated researches related to iris recognition subject.

Rai and Yadav (2014) considered a new method for recognition of iris patterns using a combination of Hamming's distance and support vector machine. The zone of the zigzag collar of the iris is selected for the extraction of iris characteristics because it captures the most important areas of the complex iris pattern and a higher recognition rate is achieved. The proposed approach also used the detection of parabolas and the cut medium filter for the detection and removal of eyelids and eyelashes, respectively. The proposed method is efficient from a computer and reliable point of view, with a recognition rate of $99.91 \%$ and $99.88 \%$ based on the image data of Cassia and Check, respectively [59].

Hamouchene et al. (2014) implemented a new iris recognition system using a new feature extraction method. The proposed method, Neighborhood-based binary pattern, compares each neighbor of the center pixel with the next neighbor to code it for 1 if it is greater than the center pixel or 0 if it is smaller than the center pixel. The resulting binary code is converted into a decimal number to build the NBP image. To deal with the problem of rotation, we propose a coding process to obtain an invariant image by rotation. This image is subdivided into several blocks and the average of each block is calculated; then, the variations of the averages are encoded by a binary code [60].

Santos et al. (2015) focused on the biometric recognition in mobile environments using iris and periocular information 
as main characteristics. This study makes three main contributions: First demonstrated the utility of an iris and a set of periocular data, which contains images acquired with 10 different mobile configurations and the corresponding data of iris segmentation. This data set allows us to evaluate iris segmentation and recognition methods, as well as periocular recognition techniques; second reported the results of device-specific calibration techniques that compensate for the different color perceptions inherent in each configuration; and third proposed the application of well-known iris and periocular recognition strategies based on classic coding and matching techniques, as well as the demonstration of how they can be combined to overcome the problems associated with mobile environments [61].

Umer et al. (2015) proposed a new set of characteristics for personal verification and identification based on iris images. The method has three main components: Image preprocessing, feature extraction, and classification. During image preprocessing, iris segmentation is performed using the Hough restricted circular transformation. Then, only two disjoint quarters of the segmented iris pattern are normalized, which allow the extraction of characteristics for classification purposes. Here, the method of extracting characteristics of an iris model is based on a morphological operator of multiple scales. Then, the characteristics of the iris are represented by the sum of the dissimilarity residues obtained by the application of a morphological top-hat transform [62].

Thomas et al. (2016) in this work, our system introduces a more accurate method called Random Sample Consensus to adjust the ellipse around the non-circular iris boundaries. You can locate the iris boundaries more accurately than the methods based on the Hough transformation. We also use the Daugman rubber sheet model for iris normalization and elliptical unpacking, and correspondence based on the correlation filter for in-class and interclass evaluation. Peak side lobe ratio is the measure of similarity used for the corresponding models. Through these, the recognition process improves with the Daugman method. The WVU database is used to perform experiments and promising results are obtained [63].

Hajari et al. (2016) showed that iris recognition is a difficult problem in a noisy environment. Their main objective is to develop a reliable iris recognition system that can operate in a noisy image environment and increase the rate of iris recognition in the CASIA and MMU iris datasets. They proposed two algorithms: First, a new method to eliminate the noise of the iris image and, second, a method to extract the characteristics of the texture through a combined approach of the local binary model and the gray level cooccurrence matrix. The proposed approach provided the highest recognition rate of $96.5 \%$ and low error rate and required less uptime [64].

Soliman et al. (2017) introduced a rough algorithm to solve the computational cost problem while achieving an acceptable precision. The gray image of the iris is transformed into a binary image using an adaptive threshold obtained from the analysis of the intensity histogram of the image. The morphological treatment is used to extract an initial central point, which is considered the initial center of the iris and pupil boundaries. Finally, a refinement step is performed using an integrodifferential operator to obtain the centers and the final rays of the iris and the pupil. This system is robust against occlusions and intensity variations [65].

Naseem et al. (2017) proposed an algorithm to compare the vanguard spatial representation classification with Bayesian fusion for several sectors. The proposed approach has shown that it overall performs the implemented algorithm in standard databases. The complexity analysis of the proposed algorithm shows a decisive superiority of the proposed approach. In this research, the concept of class-specific dictionaries for iris recognition is proposed. Essentially, the query image is represented as a linear combination of learning images of each class. The well-conditioned inverse problem is solved using the least squares regression and the decision is judged in favor of the class with the most accurate estimate [66].

Llano et al. (2018) presented a robust and optimized multisensor scheme with a strategy that combines the evaluation of video frame quality with robust segmentation fusion methods for image recognition and simultaneous image iris recognition. As part of the proposed scheme, they presented a fusion method based on the modified Laplacian pyramid in the segmentation stage. The experimental results in the Casia-V3-Interval, Casia-V4-Mile, Ubiris-V1, and MBGC-V2 databases show that the robust optimized scheme increases recognition accuracy and is robust for different types of iris sensors [67].

Zhang et al. (2018) implemented a generalized stimulation framework to solve some problems of practical recognition of the iris at a distance, namely, the detection of the iris, the detection of the poor location of the iris, the detection of iris, and iris recognition. This solution takes advantage of a set 
of carefully designed features and well-adjusted stimulation algorithms. Basically, there are two main contributions. The first is an exploration of the intrinsic properties of remote iris recognition, as well as robust features carefully designed for specific problems. The second important contribution is the methodology on how to adapt AdaBoost's learning to specific problems [68].

\section{RESEARCH METHODOLOGY}

\subsection{Iris Image Dataset}

The construction of iris image dataset is a difficult job due to many reasons such as distance, lighting, and the resolution of the used device. This research needs to collect iris images of real patients those have some diseases on their iris. The captured iris images are of 8-bit gray images with a resolution of $480 * 640$. In general, the iris is approximately form a circular shape. The diameter of the iris in the captured image in this dataset is about 200 pixels. Twenty eye images of 10 patients infected with anterior uveitis are applied in this research.

\subsection{Implemented System}

During a brief reviewing in this field, you can find many systems and algorithms are implemented for biometric recognition including iris recognition. The proposed implemented approach for iris recognition contains the following components (Fig. 2):

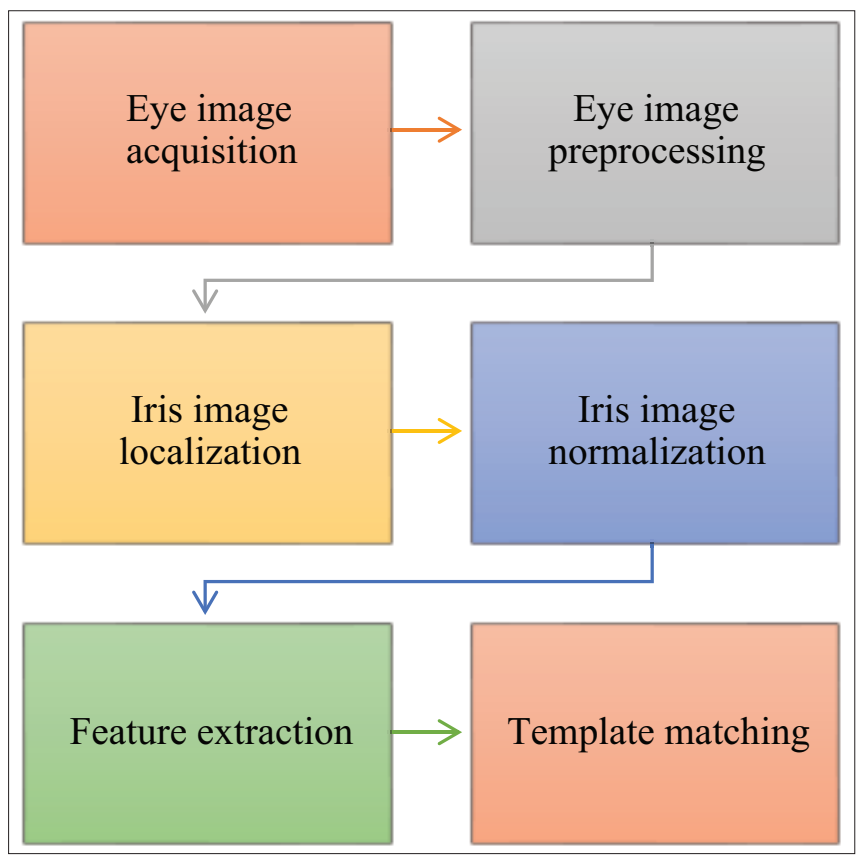

Fig. 2. Implemented approach of iris recognition.
- Eye image acquisition: In this step, the eye object is captured using sensitive device to convert the real iris object into digital image contains of number of effective pixels.

- Eye image preprocessing: In this step, the acquired digital image is converted into standard image that can be adapted for the next step of processing. This step passed into many processes such as converting the image into gray scale image, image filtering, and image resizing.

- Iris image localization: In this step, the diseased eye image is enhanced to track the iris region to detect and localize the iris region.

- Iris image normalization: In this step, the iris image is normalized and then converted into gray scale to generate a standard iris image to be adequate for the next step for processing.

- Feature extraction: This step deals with the generation of features or characteristics related to the indicated iris image. Feature is extracted using two-dimensional discrete wavelet transform (2D DWT). 2D DWT is performed through passing low-pass filter and high-pass filter for both rows and columns of the image as shown in the following two equations:

$$
\begin{aligned}
& x_{\text {low }}=\sum_{k=-N}^{N} x[k] g[2 n-k] \\
& x_{\text {bigh }}=\sum_{k=-N}^{N} x[k] b[2 n-k]
\end{aligned}
$$

Where, $\mathrm{x}$ represents the input array and both $\mathrm{g}$ and $\mathrm{h}$ represent low-pass and high-pass filters, respectively.

- Template matching: In this step, the template matching is generated that can be used to decide the personal authentication based on the selected threshold.

\subsection{Detection of Diseased Eye}

There are many differences between diseased eye and normal eye as shown in Fig. 3. One important issue is to identify the diseased eye, in which the pupil of the diseased eye with anterior uveitis is not circular and may cause changes in iris architecture or atrophy. In this study, two factors are considered to separate between diseased and normal eyes:

- To localize the pupil boundary or iris boundary as a circle, its radius must fall within the specific range. In the specified database, the range of iris radius value is within 90-150 pixels, while the pupil radius ranges are within 28-75 pixels.

- The pupil is always within the iris region; hence, the pupil boundary must be within the iris boundary for normal 
eyes, while in the diseased eyes, the pupil boundary is localized away from iris region. This gives an evidence that the eye is diseased and an enhancement must be introduced before iris localization step.

\subsection{Enhancement of Iris Image}

When decision is taken that the eye is infected or diseased, then the procedure goes directly to the enhancement process. The enhancement process helps to localize the pupil boundary. The enhancement process is implemented through the following steps:

1. Determine the upper boundary of iris, which leads to cover the area within the pupil (Fig. 4). At this process, three parameters are stored: The upper radius $\left(R_{\text {upper }}\right)$, $X_{\text {upper }}$ boundary of the iris $\left(X_{\text {upper }}\right)$, and $Y_{\text {upper }}$ boundary of the iris $\left(Y_{\text {upper }}\right)$.

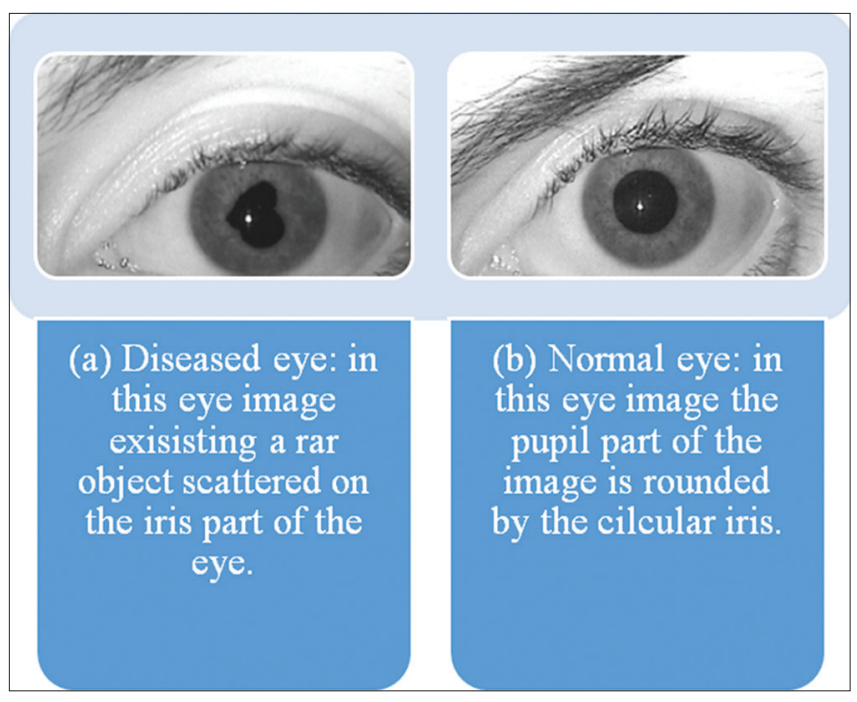

Fig. 3. Diseased and normal eyes.

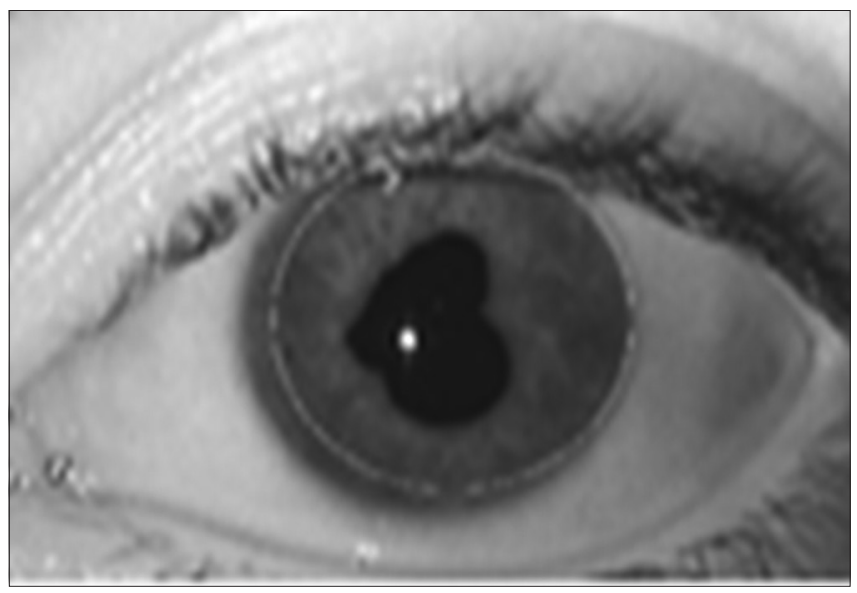

Fig. 4. Iris boundary localization.
2. Resizing the eye image to isolate the iris image as shown in Fig. 5. The pupil boundary will be localized within the iris region instead of the whole eye region. Hence, the new iris image will be determined as below:

$P_{1}=\left(X_{\text {upper }}-R_{\text {upper }}, Y_{\text {upper }}-R_{\text {upper }}\right)$

$P_{2}=\left(X_{\text {upper }}^{\text {upper }}+R_{\text {upper }}, Y_{\text {upper }}^{\text {upper }}+R_{\text {upper }}\right)$

3. Adjusting the intensity of iris image according to the incident light as shown in Fig. 6.

4. Adjusting the threshold value to create the binary image as shown in Fig. 7.

5. Discriminate the irregular pupil by determining the minimum and maximum points on each axis as shown in Fig. 8. Four points must be calculated in this step: $P_{x \text { min }}$ refers to the minimum point on $X$-axis found in pupilpixels $P_{y \text { min }}$ refers to the minimum point on $Y$-axis found in pupil pixels

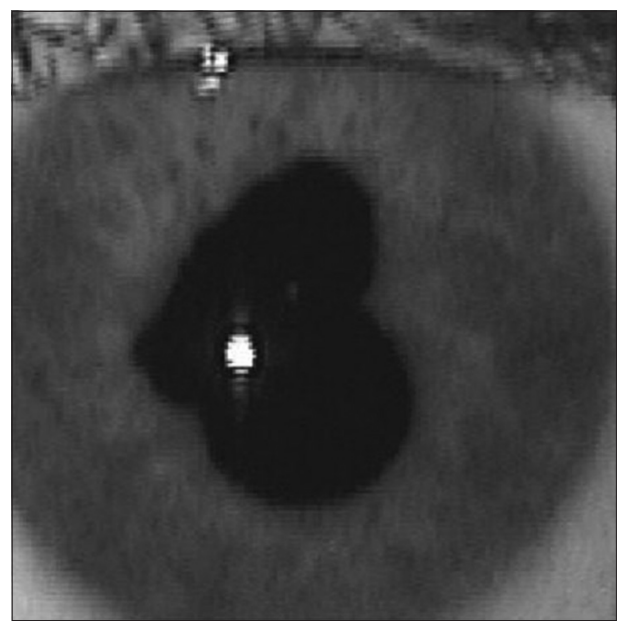

Fig. 5. Iris resizing.

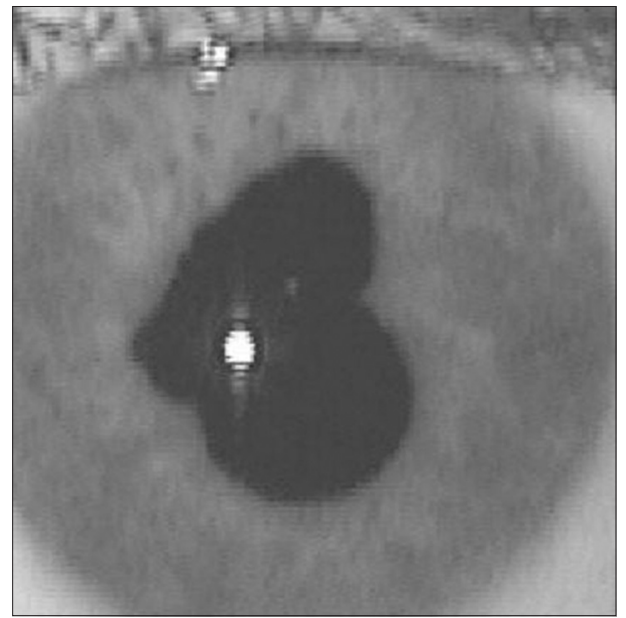

Fig. 6. Adjusting iris image.

UHD Journal of Science and Technology | Jul 2019 | Vol 3 | Issue 2 
$P_{x \text { max }}$ refers to the maximum point on $X$-axis found in pupil pixels

$P_{y \text { max }}$ refers to the maximum point on $Y$-axis found in pupil pixels

(8)

6. Round the irregular pupil area by a rectangular shape as shown in Fig. 9. This rectangular shape contains all the pixels of the irregular pupil. Two points must be calculated in this step:

$P_{1 \text { min }}=\left(X_{\text {px min }}, Y_{\text {py min }}\right)$

$P_{1 \text { max }}=\left(X_{p x \max }, Y_{p y \text { max }}\right)$

7. Calculate the center of the rectangular according to the previous step:

$P_{\text {center }}=\left(X_{\text {center }}, Y_{\text {center }}\right)$

8. Draw a circle around the pupil to complete the circular form of the pupil as shown in Fig. 10.

9. Update the iris image to the same position on the original image.

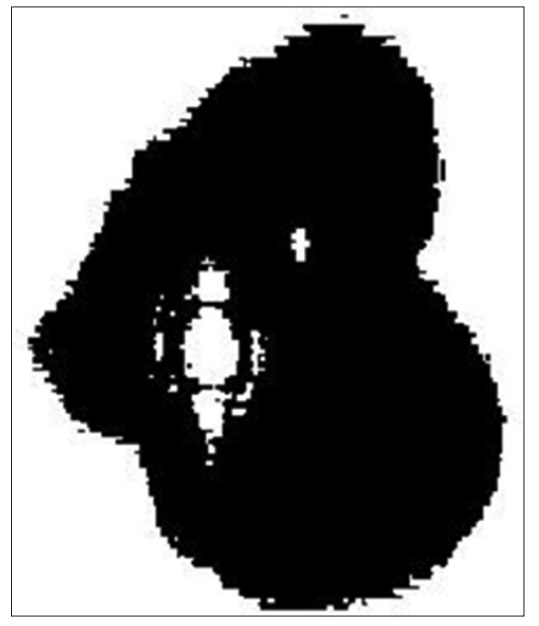

Fig. 7. Thresholding of iris image.

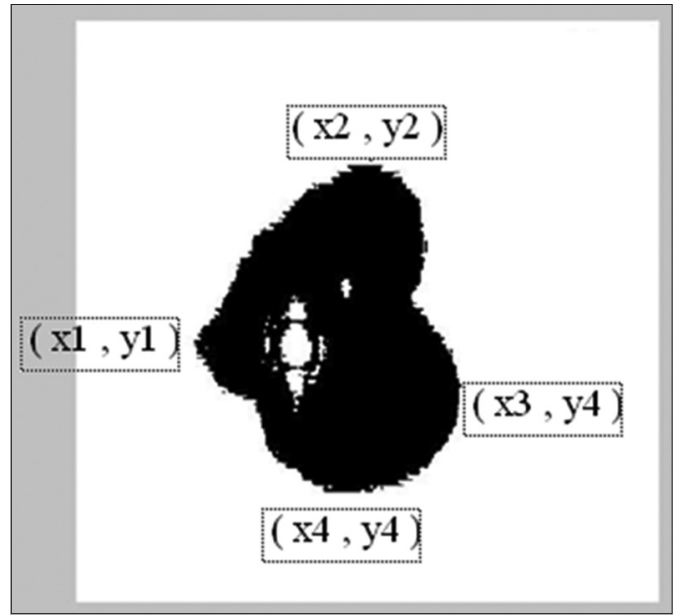

Fig. 8. Determine rectangular dimensions.
10. Compare the processed image with the images stored in the database to identify the person.

\section{RESULTS AND DISCUSSION}

Hamming distance measures the fraction of disagreeing bits resulting from bit-by-bit comparison of the two regions of interest. The obtained result indicated that the criterion is chosen to be 0.40 , which means that a matching decision is never declared between two iris codes if it is exceed $40 \%$ of the disagreed bits. Fig. 11 illustrates that the change in Hamming distance before and after applying the proposed approach; in addition, it is clear that applying this approach causes significant decreasing in the Hamming distance value.

Fig. 12 illustrates a comparison between the Hamming distance of the diseased eye images before and after treatment

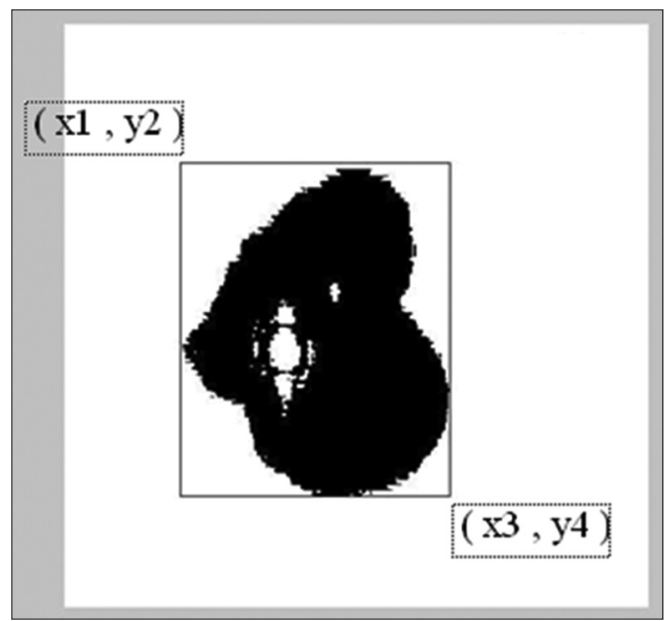

Fig. 9. Round the irregular pupil area by a rectangular shape.

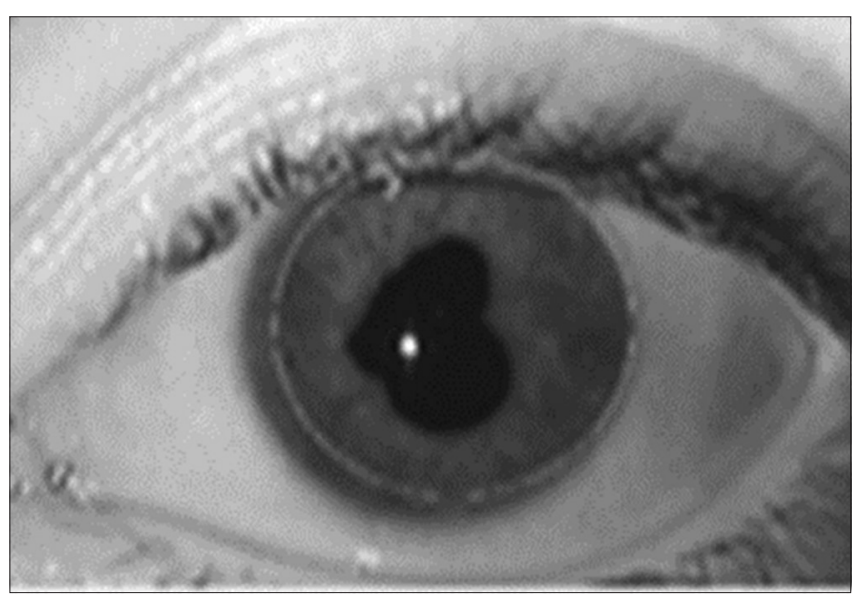

Fig. 10. Drawing a circle around the pupil. 


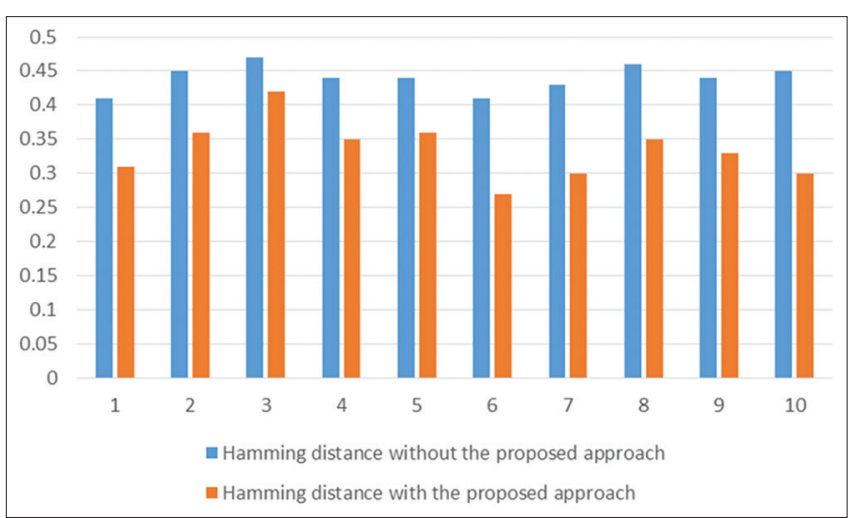

Fig. 11. Change in Hamming distance before and after applying the proposed approach.

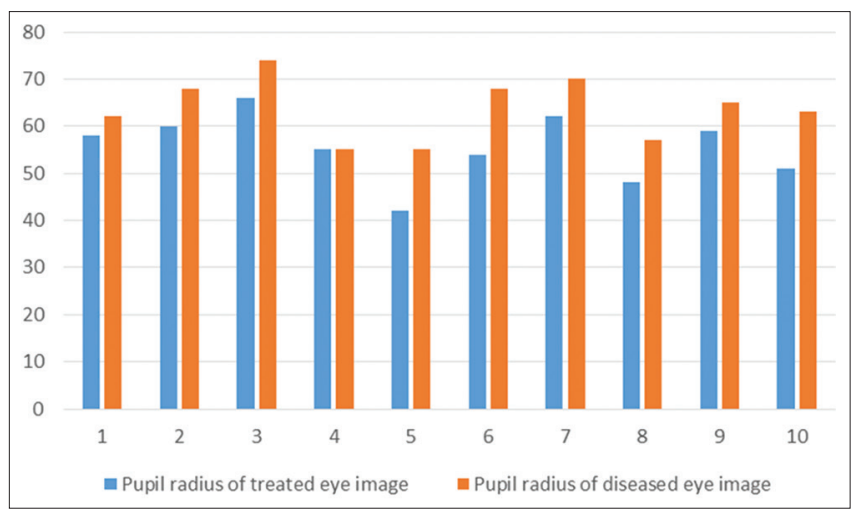

Fig. 12. The Hamming distance outcomes of the diseased eye images before and after treatment and the Hamming distance outcomes for the images of the same diseased eye after treatment.

and the Hamming distance for the images of the same diseased eye after treatment. This figure indicated that the Hamming distance values for the two diseased eye images after treatment are less than the Hamming distance values for the images of the diseased eye before and after treatment. These results are caused by the pupil of the treated eyes seem normal unlike diseased eyes whose pupil becomes a little larger after applying the proposed enhancement method.

Fig. 13 indicated the differences in pupil radius values between the images of the diseased eyes after applying the enhancement and the images of the same diseased eyes after receiving the processed image. According to pupil distortion in the diseased eyes, the size of the pupil will enlarge affecting the size of iris region which should be considered when calculating Hamming distance in iris recognition algorithm; this caused increasing the Hamming distance values.

The implemented approach is evaluated through performance evaluation in terms of false rejection rate (FRR) and false

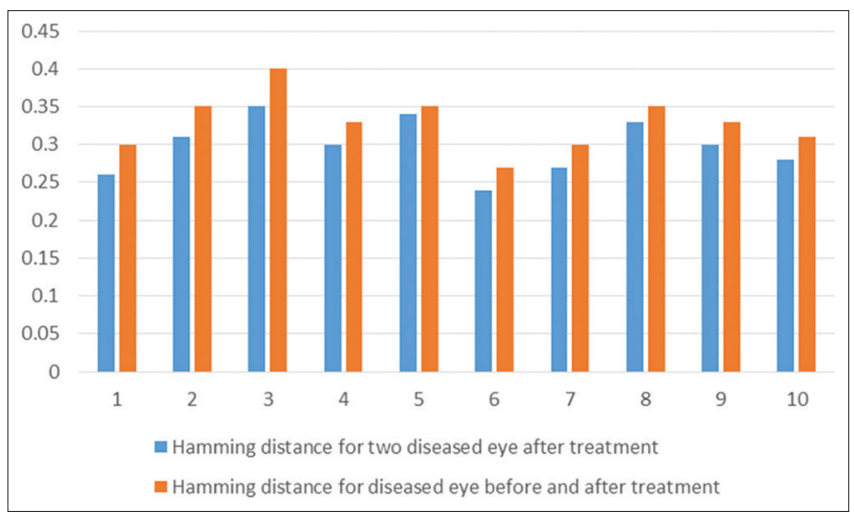

Fig. 13. Pupil radius values of diseased and treated eyes.

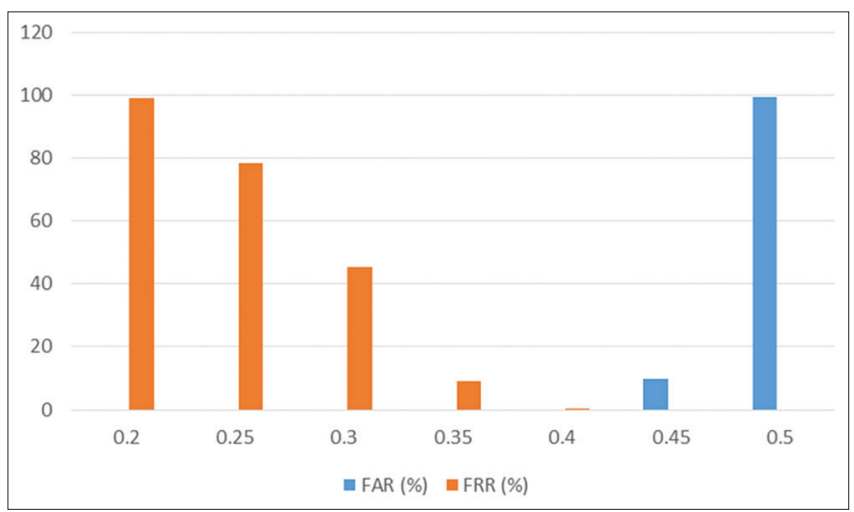

Fig. 14. Performance evaluation in terms of false rejection rate and false acceptance rate.

acceptance rate (FAR). FAR and FRR are shown in Fig. 14 based on the Hamming distance. Perfect recognition is not possible due to the overlapping distributions. An accurate recognition rate is achieved through threshold of 0.40 , in which a false accept rate and false reject rate of $0.000 \%$ and $0.100 \%$, respectively, are obtained.

\section{CONCLUSIONS}

Iris recognition is an effective method for biometric human identification. The implemented iris recognition approach is passed into many steps to achieve good system performance. This research studied the effects of infected eye on the recognition process through introducing different types of eye images. In addition, treating and enhancing processes are inserted in the overall approach to prepare an adequate iris image for processing. The obtained results indicated that the recognition performance of the implemented approach is $90 \%$. The experimental results show that the proposed method is an effective approach in iris recognition. 


\section{REFERENCES}

[1] M. S. Al-Ani. Biometric Security, Source Title: Handbook of Research on Threat Detection and Countermeasures in Network Security. IGI Global, Pennsylvania (USA), 2015.

[2] A. E. Osborn-Gustavson, T. McMahon, M. Josserand and B. J. Spamer. The utilization of databases for the identification of human remains. In: New Perspectives in Forensic Human Skeletal Identification. Ch. 12. Academic Press, San Diego, 2018, pp. 129139.

[3] M. S. Al-Ani. "Happiness measurement via classroom based on face tracking. UHD Journal of Science and Technology, vol 3, no. 1, pp. 9-17, 2019.

[4] M. Viner. Overview of advances in forensic radiological methods of human identification. In: New Perspectives in Forensic Human Skeletal Identification. Ch. 19. Academic Press, San Diego, 2018, pp. 217-226.

[5] M. S. Al-Ani. Biometrics: Identification and security, source title. In: Multidisciplinary Perspectives in Cryptology and Information Security. IGI Global, Pennsylvania (USA), 2014.

[6] S. S. Muhamed and M. S. Al-Ani. "Signature recognition based on discrete wavelet transform". UHD Journal of Science and Technology, vol. 3, no. 1, pp. 19-29, 2019.

[7] A. M. Christensen and G. M. Hatch. Advances in the use of frontal sinuses for human identification. In: New Perspectives in Forensic Human Skeletal Identification. Ch. 20, Academic Press, San Diego, 2018, pp. 227-240.

[8] M. S. Al-Ani and K. M. Ali Alheeti. Precision statistical analysis of images based on brightness distribution. Advances in Science, Technology and Engineering Systems Journal, vol. 2, no. 4, pp. 99-104, 2017.

[9] M. S. Al-Ani. Efficient architecture for digital image processing based on EPLD. IOSR Journal of Electrical and Electronics Engineering, vol. 12, no. 6, pp. 1-7, 2017.

[10] M. S. Al-Ani, T. N. Muhamad, H. A. Muhamad and A. A. Nuri. Effective Fingerprint Recognition Approach Based on Double Fingerprint Thumb, 2017 IEEE, 2017 International Conference on Current Research in Computer Science and Information Technology (ICCIT). IEEE, Slemani-Iraq, 2017.

[11] J. L Cambier. Adaptive iris capture in the field. Biometric Technology Today, vol. 2014, no. 2, pp. 5-7, 2014.

[12] A. Rodriguez and B. V. K. Vijaya Kumar. Segmentation-free biometric recognition using correlation filters. Academic Press Library in Signal Processing. Ch. 15. Vol 4. Carnegie Mellon University, Pittsburgh, PA, USA, 2014, pp. 403-460.

[13] P. Tome, R. Vera-Rodriguez, J. Fierrez and J. Ortega-Garcia. Facial soft biometric features for forensic face recognition. Forensic Science International, vol. 257,, pp. 271-284, 2015.

[14] M. S. Nixon, P. L. Correia, K. Nasrollahi, T. B. Moeslund and M. Tistarelli. On soft biometrics. Pattern Recognition Letters, vol. 68, pp. 218-230, 2015.

[15] I. Rigas and O. V. Komogortsev. Eye movement-driven defense against iris print-attacks. Pattern Recognition Letters, vol. 68, pp. 316-326, 2015.

[16] F. Davoodi, H. Hassanzadeh, S. A. Zolfaghari, G. Havenith and M. Maerefat. A new individualized thermoregulatory bio-heat model for evaluating the effects of personal characteristics on human body thermal response. Building and Environment, vol. 136, pp. 62-76, 2018.
[17] P. Connor and A. Ross. Biometric recognition by gait: A survey of modalities and features. Computer Vision and Image Understanding, vol. 167, pp. 1-27, 2018.

[18] K. Nguyen, C. Fookes, S. Sridharan, M. Tistarelli and M. Nixon. Super-resolution for biometrics: A comprehensive survey. Pattern Recognition, vol. 78, pp. 23-42, 2018.

[19] G. Batchuluun, J. H. Kim, H. G. Hong, J. K. Kang and K. R. Park. Fuzzy system based human behavior recognition by combining behavior prediction and recognition. Expert Systems with Applications, vol. 81, pp. 108-133, 2017.

[20] S. Gold. Iris biometrics: A legal invasion of privacy? Biometric Technology Today, vol. 2013, no. 3, pp. 5-8, 2013.

[21] M. Gomez-Barrero, J. Galbally and J. Fierrez. Efficient software attack to multimodal biometric systems and its application to face and iris fusion. Pattern Recognition Letters, vol. 36, pp. 243-253, 2014.

[22] K. Aloui, A. Nait-Ali and M. S. Naceur. Using brain prints as new biometric feature for human recognition. Pattern Recognition Letters, vol. 113, In Press, 2017.

[23] S. Kumar and S. K. Singh. Monitoring of pet animal in smart cities using animal biometrics. Future Generation Computer Systems, vol. 83, pp. 553-63, 2018.

[24] S. Crihalmeanu and A. Ross. Multispectral scleral patterns for ocular biometric recognition. Pattern Recognition Letters, vol. 33, no. 14, pp. 1860-1869, 2012.

[25] K. C. Reshmi, P. I. Muhammed, V. V. Priya and V. A. Akhila. A novel approach to brain biometric user recognition. Procedia Technology, vol. 25, pp. 240-247, 2016.

[26] H. Wechsler and F. Li. Biometrics and robust face recognition. In: Conformal Prediction for Reliable Machine Learning. Ch. 10. Morgan Kaufmann Publishers Inc., San Francisco, 2014, pp. 189215.

[27] M. S. Al-Ani and Q. Al-Shayea. Speaker identification: A novel fusion samples approach. International Journal of Computer Science and Information Security, vol. 14, no. 7, pp. 423-427, 2016.

[28] R. S. Prasad, M. S. Al-Ani and S. M. Nejres. Hybrid fusion of two human biometric features. International Journal of Business and ICT, vol. 2, pp. 1-2, 2016.

[29] Q. Al-Shayea and M. S. Al-Ani. Biometric face recognition based on enhanced histogram approach. International Journal of Communication Networks and Information Security, vol. 10, no. 1, pp. 148-154, 2018.

[30] T. Bergmüller, E. Christopoulos, K. Fehrenbach, M. Schnöll and A. Uhl. Recompression effects in iris recognition. Image and Vision Computing, vol. 58, pp. 142-157, 2017.

[31] M. Trokielewicz, A. Czajka and P. Maciejewicz. Implications of ocular pathologies for iris recognition reliability. Image and Vision Computing, vol. 58, pp. 158-167, 2017.

[32] Y. Alvarez-Betancourt and M. Garcia-Silvente. A keypoints-based feature extraction method for iris recognition under variable image quality conditions. Knowledge-Based Systems, vol. 92, pp. 169182, 2016.

[33] A. K Bhateja, S. Sharma, S. Chaudhury and N. Agrawal. Iris recognition based on sparse representation and k-nearest subspace with genetic algorithm. Pattern Recognition Letters, vol. 73, pp. 13-18, 2016.

[34] R. Pasula, S. Crihalmeanu and A. Ross. A multiscale sequential fusion approach for handling pupil dilation in iris recognition. 
In: Human Recognition in Unconstrained Environments. Ch. 4. Academic Press, Chicago, IL, 2017, pp. 77-102.

[35] F. Jan. Segmentation and localization schemes for non-ideal iris biometric systems. Signal Processing, vol. 133, pp. 192-212, 2017.

[36] D. Gragnaniello, G. Poggi, C. Sansone and L. Verdoliva. Using iris and sclera for detection and classification of contact lenses. Pattern Recognition Letters, vol. 82, pp. 251-257, 2016.

[37] Y. Hu, K. Sirlantzis and G. Howells. Iris liveness detection using regional features. Pattern Recognition Letters, vol. 82, pp. 242250, 2016.

[38] M. De Marsico, C. Galdi, M. Nappi and D. Riccio. FIRME: Face and iris recognition for mobile engagement. Image and Vision Computing, vol. 32, no. 12, pp. 1161-1172, 2014.

[39] J. Liu, Z. Sun and T. Tan. Distance metric learning for recognizing low-resolution iris images. Neurocomputing, vol. 144, pp. 484-492, 2014.

[40] R. S. Prasad, M. S. Al-Ani and S. M. Nejres. Human identification via face recognition: Comparative study. IOSR Journal of Computer Engineering, vol. 19, no. 3, pp. 17-22, 2017.

[41] G. I. Raho, M. S. Al-Ani, A. A. K. Al-Alosi and L. A. Mohammed. Signature recognition using discrete fourier transform. International Journal of Business and ICT, vol. 1, pp. 1-2, 2015.

[42] R. S. Prasad, M. S. Al-Ani and S. M. Nejres. An efficient approach for human face recognition. International Journal of Advanced Research in Computer Science and Software Engineering, vol. 5, no. 9, pp. 133-136, 2015.

[43] R. S. Prasad, M. S. Al-Ani and S. M. Nejres. An efficient approach for fingerprint recognition. International Journal of Engineering Innovation and Research, vol. 4, no. 2, pp. 303-313, 2015.

[44] K. Nguyen, C. Fookes, R. Jillela, S. Sridharan and A. Ross. Long range iris recognition: A survey. Pattern Recognition, vol. 72, pp. 123-143, 2017.

[45] M. Karakaya. A study of how gaze angle affects the performance of iris recognition. Pattern Recognition Letters, vol. 82, pp. 132-143, 2016.

[46] K. B. Raja, R. Raghavendra, V. K. Vemuri and C. Busch. Smartphone based visible iris recognition using deep sparse filtering. Pattern Recognition Letters, vol. 57, pp. 33-42, 2016.

[47] K. W. Bowyer, E. Ortiz and A. Sgroi. Iris recognition technology evaluated for voter registration in Somaliland. Biometric Technology Today, vol. 2015, no. 2, pp. 5-8, 2015.

[48] A. F. M. Raffei, H. Asmuni, R. Hassan and R. M. Othman. A low lighting or contrast ratio visible iris recognition using iso-contrast limited adaptive histogram equalization. Knowledge-Based Systems, vol. 74, pp. 40-48, 2015.

[49] SwathiS. Dhage, S. S. Hegde, K. Manikantan and S. Ramachandran. DWT-based feature extraction and radon transform based contrast enhancement for improved iris recognition. Procedia Computer Science, vol. 45, pp. 256-265, 2015.

[50] S. Umer, B. C. Dhara and B. Chanda. A novel cancelable iris recognition system based on feature learning techniques. Information Sciences, vol. 406-407, pp. 102-118, 2015.

[51] Y. Jung, D. Kim, B. Son and J. Kim. An eye detection method robust to eyeglasses for mobile iris recognition. Expert Systems with Applications, vol. 67, pp. 178-188, 2017.

[52] I. Tomeo-Reyes and V. Chandran. Part based bit error analysis of iris codes. Pattern Recognition, vol. 60, pp. 306-317, 2016.

[53] Haiqing Li, Q. Zhang and Z. Sun. Iris recognition on mobile devices using near-infrared images. In: Human Recognition in Unconstrained Environments. Ch. 5. Institute of Automation, Chinese Academy of Sciences, Beijing, PR China, 2017, pp. 103117.

[54] S. S. Barpanda, B. Majhi, P. K. Sa, A. K. Sangaiah and S. Bakshi. Iris feature extraction through wavelet mel-frequency cepstrum coefficients. Optics and Laser Technology, vol. 110, pp. 13-23, 2019.

[55] M. Sardar, S. Mitra and B. U. Shankar. Iris localization using rough entropy and CSA: A soft computing approach. Applied Soft Computing, vol. 67, pp. 61-69, 2018.

[56] S. Zhang and Y. Zhou. Template matching using grey wolf optimizer with lateral inhibition. Optik-International Journal for Light and Electron Optics, vol. 130, pp. 1229-1243, 2017.

[57] P. Samant and R. Agarwal. Machine learning techniques for medical diagnosis of diabetes using iris images. Computer Methods and Programs in Biomedicine, vol. 157, pp. 121-128, 2018.

[58] Z. Lin, D. Ma, J. Meng and L. Chen. Relative ordering learning in spiking neural network for pattern recognition. Neurocomputing, vol. 275, pp. 94-106, 2018.

[59] H. Rai and A. Yadav. Iris recognition using combined support vector machine and Hamming distance approach. Expert Systems with Applications, vol. 41, no. 2, pp. 588-593, 2014.

[60] I. Hamouchene and S. Aouat. A new texture analysis approach for iris recognition. AASRI Procedia, vol. 9, pp. 2-7, 2014.

[61] G. Santos, E. Grancho, M. V. Bernardo and P. T. Fiadeiro. Fusing iris and periocular information for cross-sensor recognition. Pattern Recognition Letters, vol. 57, pp. 52-59, 2015.

[62] S. Umer, B. C. Dhara and B. Chanda. Iris recognition using multiscale morphologic features. Pattern Recognition Letters, vol. 65, pp. 67-74, 2015.

[63] T. Thomas, A. George and K. P. I. Devi. Effective iris recognition system. Procedia Technology, vol. 25, pp. 464-472, 2016.

[64] K. Hajari, U. Gawande and Y. Golhar. Neural network approach to iris recognition in noisy environment. Procedia Computer Science, vol. 78, pp. 675-682, 2016.

[65] N. F. Soliman, E. Mohamed, F. Magdi, F. E. A. El-Samie and A. M. Elnaby. Efficient iris localization and recognition. Optik-International Journal for Light and Electron Optics, vol. 140, pp. 469-475, 2017.

[66] I. Naseem, A. Aleem, R. Togneri and M. Bennamoun. Iris recognition using class-specific dictionaries. Computers and Electrical Engineering, vol. 62, pp. 178-193, 2017.

[67] E. G. Llano, M. S. G. Vázquez, J. M. C. Vargas, L. M. Z. Fuentes and A. A. R. Acosta. Optimized robust multi-sensor scheme for simultaneous video and image iris recognition. Pattern Recognition Letters, vol. 101, pp. 44-51, 2018.

[68] M. Zhang, Z. He, H. Zhang, T. Tan and Z. Sun. Towards practical remote iris recognition: A boosting based framework. Neurocomputing, vol. 330, In Press, 2018. 